\title{
Caracterización de minerales pesados en sedimentos activos de la cuenca del río Magdalena, Colombia: implicaciones para el análisis de procedencia en el registro fluvial
}

\author{
Felipe Velásquez $^{1 *}$, Agustín Cardona ${ }^{2,3}$, Camilo Montes ${ }^{4}$
}

DOI: http://dx.doi.org/10.18273/revbol.v41n2-2019008@ @ (1)

Forma de citar: Velásquez, F., Cardona, A., y Montes, C. (2019). Caracterización de minerales pesados en sedimentos activos de la cuenca del río Magdalena, Colombia: implicaciones para el análisis de procedencia en el registro fluvial. Boletín de Geología, 41(2), 137-147. DOI: 10.18273/revbol.v41n2-2019008.

\section{RESUMEN}

La cuenca del río Magdalena, ubicada en los Andes colombianos, es sin duda la más importante del país, con un área de 257.400 $\mathrm{km}^{2}$ y altitudes entre 0 a $5.670 \mathrm{msnm}$. Esta cuenca se caracteriza por tener una gran variedad de unidades litológicas a lo largo de una topografía abrupta e intensos procesos de erosión y transporte, generando sedimentos recientes con una alta concentración de minerales. En el presente trabajo se caracterizaron los minerales pesados de 21 muestras de arenas en barras de canal del río Magdalena y algunos de sus ríos tributarios de primer y segundo orden hacia el oriente y occidente de la cuenca. La zona occidental de la cuenca del Magdalena mostró un predominio de minerales inestables como olivino, piroxeno, anfíbol y minerales del grupo de la epidota con buen estado de conservación, procedentes de complejos ígneos y metamórficos de las Cordilleras Central y Occidental, mientras que la zona oriental de la cuenca mostró un predominio de minerales ultraestables de circón y turmalina, procedentes de secuencias sedimentarias de la Cordillera Oriental. Por otra parte, el río Magdalena presenta un patrón de homogenización, mezclando la proporción de minerales inestables, metaestables y ultraestables de sus diferentes ríos tributarios. Los resultados confirman que, a escala regional los minerales pesados procedentes de drenajes de primer y segundo orden son altamente diagnósticos de la geología del área fuente, mientras que los drenajes de tercer orden por su carácter mixto, tienden a obliterar las proporciones de minerales pesados, siendo por lo tanto menos diagnósticos en términos de procedencia. Adicionalmente, la preservación de fases minerales altamente inestables en las muestras analizadas, sugiere que en los climas tropicales la topografía juega un papel fundamental en la eficiencia de la meteorización y la preservación de especies minerales.

Palabras clave: Río Magdalena; minerales pesados; arenas recientes; Andes del Norte; procedencia.

\section{Characterization of heavy minerals from active sediments of the Magdalena River basin, Colombia: implications in the provenance analysis of the fluvial record}

\begin{abstract}
The Magdalena River basin, located on the Colombian Andes, is the most important basin in the country, with an area of 257,400 $\mathrm{km}^{2}$ and altitudes between 0 to 5,670 $\mathrm{m}$. The basin is characterized by having a huge variety of lithological units along an abrupt topography and intense processes of erosion and transport, generating recent sediments with a high concentration of minerals. In the present work, the heavy minerals of 21 recent sand samples from channel bars of the Magdalena River and different first and second order tributary drainages towards the east and west of the basin were analyzed. Drainage from the Central and Western Cordillera are characterized by abundant and well-preserved unstable minerals such as olivine, pyroxene, amphibole and minerals of the epidote group, that have their source areas in the different igneous and metamorphic complexes from this Cordilleras. Drainage derived in the eastern Cordillera showed a predominance of ultrastable zircon and tourmaline minerals, coming from sedimentary sequences. On the other hand, the Magdalena River is characterized by a mixed proportion of unstable, metastable and ultrastable minerals of its different tributary rivers. The results confirm that on a regional scale, heavy minerals from first and second order drainages are highly diagnostic of the geology of the source area while third order and regional scales drainages, due to their mixed nature, tend to obliterate the proportions of heavy minerals, being therefore less diagnostic in terms of provenance. Additionally, the preservation of highly unstable mineral phases in the analyzed samples, suggests that in tropical climates, the topography plays a fundamental role in the efficiency of weathering and the preservation of mineral species.
\end{abstract}

Keywords: Magdalena River; heavy minerals; recent sands; northern Andes; provenance.

\footnotetext{
${ }^{1}$ Departamento de Geociencias y Medio Ambiente, Facultad de Minas, Universidad Nacional de Colombia, Medellín, Colombia. (*) fevelasquezru@gmail.com

2 Departamento de Procesos y Energía, Facultad de Minas, Universidad Nacional de Colombia, Medellín, Colombia. agcardonamo@unal.edu.co

${ }^{3}$ Grupo de Investigación en Geología y Geofísica, Universidad Nacional de Colombia, Medellín, Colombia.

${ }^{4}$ Departamento de Física y Geociencias, Universidad del Norte, Barranquilla, Colombia. camilomontes@uninorte.edu.co
} 


\section{INTRODUCCIÓN}

El estudio sistemático de minerales pesados es una valiosa herramienta para el análisis de procedencia en secuencias antiguas y depósitos modernos, ya que estos se encuentran asociados a rocas formadas en condiciones específicas, lo cual facilita una identificación más precisa del área fuente (Garzanti y Andò, 2007a). Cuando se analiza el registro de minerales pesados en sedimentos activos, donde las características del transporte, el orden de drenaje, la meteorización y el relieve pueden conocerse, resulta posible evaluar el peso de las diferentes variables que controlan el comportamiento de los minerales pesados en los ambientes sedimentarios y los alcances de esta herramienta para el análisis de procedencia en secuencias sedimentarias antiguas.

Por la presencia de altos contenidos de Fe y Mg, las rocas máficas concentran una gran cantidad de minerales pesados (olivino, piroxeno y anfíbol), los cuales son altamente susceptibles a procesos de meteorización química, llegando a ser disueltos (Andò et al., 2012). En contraste las rocas félsicas, si bien concentran menor proporción de minerales pesados, estos pueden tener una mayor resistencia a procesos de meteorización, como es el caso de circón, la turmalina y el rutilo (Garzanti y Andò, 2007b), pudiendo experimentar múltiples ciclos sedimentarios. Así, los minerales pesados pueden ser altamente diagnósticos de la geología del área fuente. Por otra parte, el estudio de procedencia es sensible a variables como el clima, la topografía o las dimensiones de la red de drenaje (Ingersoll et al., 1993), debido a que en la meteorización de una partícula mineral, desde su exposición en el área fuente hasta su historia de transporte (p.e. exposiciones subaereas en barras de río o en deltas cuando hay cambios en el nivel del mar), los cristales presentan selección por densidad, en el fluido que transporta las partículas (Morton y Hallsworth, 1999).

La cuenca del río Magdalena ubicada en los Andes colombianos (FIGURA 1), cuenta con un área de $257.400 \mathrm{~km}^{2}$ y altitudes entre 0 a $5.670 \mathrm{msnm}$. Esta cuenca ocupa el $24 \%$ del territorio colombiano y hospeda el $80 \%$ de la población colombiana, siendo así la principal arteria fluvial del país (Restrepo, 2006; IDEAM, 2001). Geológicamente las cordilleras que la limitan, se caracterizan por una gran variedad de unidades litológicas (TABLA 1), con presencia de complejos ígneos, metamórficos y espesas secuencias sedimentarias a lo largo de una topografía abrupta donde tienen lugar múltiples procesos de erosión y transporte. En el presente trabajo se caracterizaron 21 sedimentos activos, específicamente de barras de canal del río Magdalena y algunos de sus principales ríos tributarios entre Neiva y Bocas de Ceniza, en ríos que drenan las Cordilleras Occidental, Central y Oriental y los Macizos de Perijá y Santa Marta. El objetivo de investigación es caracterizar los minerales pesados de los sedimentos recientes obtenidos en barras de canal del río Magdalena y algunos de los ríos tributarios y determinar si efectivamente estos son diagnósticos en términos de procedencia a escala regional, y si la meteorización y el relieve efectivamente son factores que pueden afectar la composición (Sevastjanova et al., 2012).

\section{Cuenca del río Magdalena}

La cuenca del río Magdalena (FIGURA 1), ubicada en los Andes Septentrionales, cuenta con un área de drenaje de $257.400 \mathrm{~km}^{2}$, se extiende por 1.612 $\mathrm{km}$ y tiene un caudal promedio anual de $7.139 \mathrm{~m}^{3} \mathrm{~s}^{-1}$ (Restrepo, 2006). Debido a su extensa longitud y a su variedad de ecosistemas, entre otros, se subdivide de Sur a Norte en valle superior, medio e inferior. Sus principales ríos tributarios aportan agua dulce para el 80\% de la población nacional (IDEAM, 2001) y son utilizados para actividades agrícolas e industriales. Por otra parte, las lluvias presentan variaciones locales en el régimen de precipitaciones, donde las zonas más secas corresponden al alto Magdalena y a la llanura del Caribe y las mayores precipitaciones ocurren en el medio Magdalena y bajo Cauca (Leyva, 2001). De acuerdo a su caudal y al área de su cuenca, los principales ríos tributarios son: el río Cauca y río San Jorge en las Cordilleras Occidental y Central; río Sumapaz, Chicamocha, Suarez, Sogamoso y Lebrija en la Cordillera Oriental y los ríos Cesar y Ranchería hacia el Norte en los Macizos de Perijá y Santa Marta. Estudios de procedencia utilizando minerales pesados han sido previamente realizados en la cuenca del Magdalena e incluyen el trabajo de Riezebos (1979), ejecutado en arenas muestreadas en barras de canal a lo largo del río Magdalena, mostró diferentes tendencias de abundancia mineral, concluyendo así que los cambios en la mineralogía se dan principalmente en función del aporte del área fuente por medio de sus ríos tributarios.

Nie et al. (2012) presentan un análisis de minerales pesados asociados a los afluentes del sinclinal Nuevo Mundo en el Valle Medio del Magdalena, documentando como la Cordillera Oriental tiene una asociación de minerales pesados y de geocronología detrítica U-Pb en circón característica. Pepper et al. (2016) también analiza circones detríticos por las 
técnicas U-Pb e isótopos de $\mathrm{Hf}$ en el río Magdalena y algunos afluentes mayores como el Cauca y el Sinú, estableciendo las áreas magmáticas más importantes y su afinidad isotópica, con el fin de comprender los procesos de generación o reciclaje de corteza en el registro Andino.

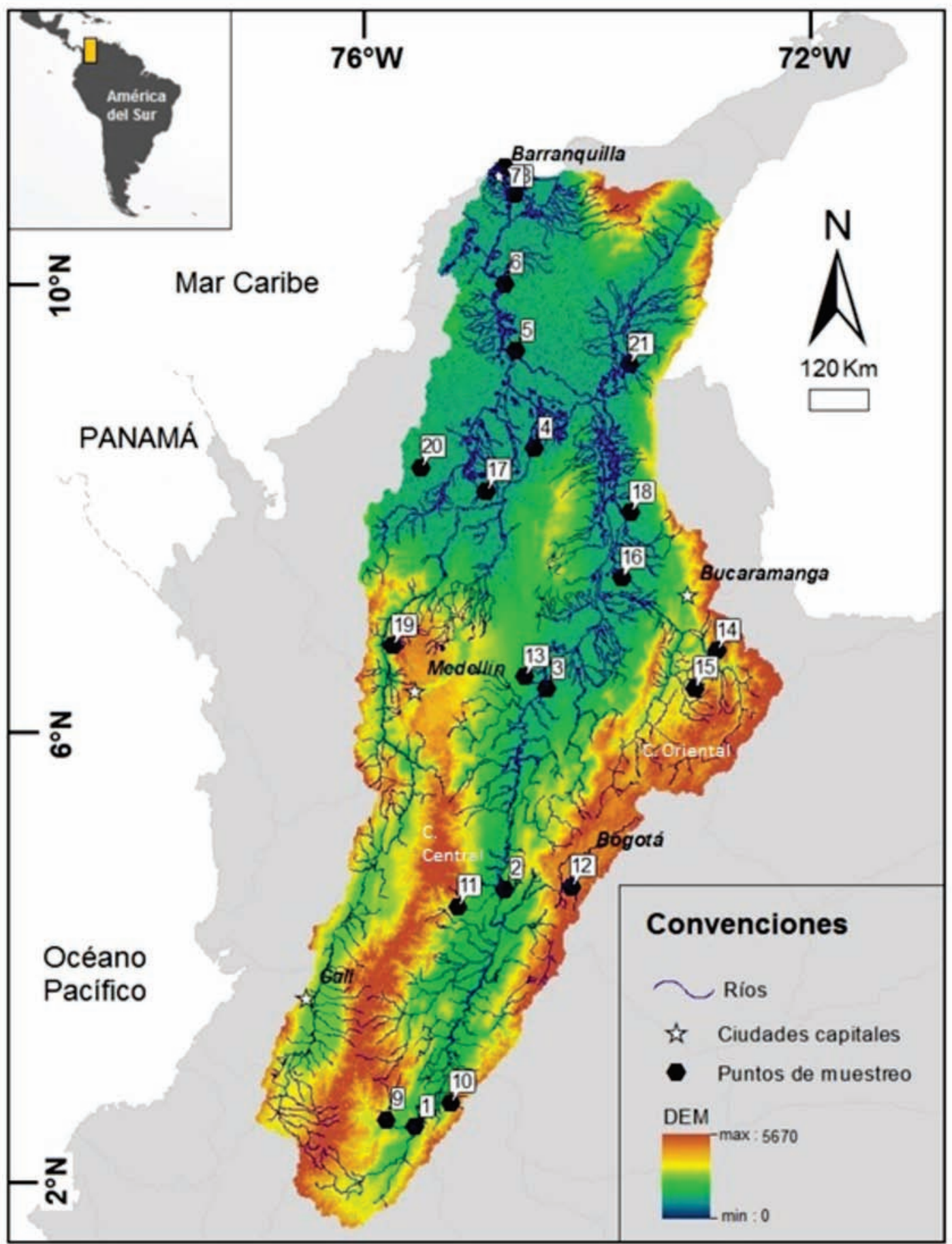

FIGURA 1. Esquema de la cuenca del río Magdalena y modelo de elevación digital (DEM) con la localización de los puntos de muestreo. 1, 2, 3, 4, 5, 6, 7 y 8: río Magdalena; 9 río Páez; 10 río Neiva; 11: río Coello; 12: río Sumapaz; 13: río Nus; 14: río Chicamocha; 15: río Suarez; 16: río Sogamoso; 17: río Cauca 01; 18: río Lebrija; 19 río Cauca 02; 20: río San Jorge; 21: río Cesar. 
TABLA 1. Área en $\mathrm{km}^{2}$ del tipo de roca en la cuenca del río Magdalena y sus ríos tributarios, calculadas por medio de ArcGIS (ESRI, 2011).

\begin{tabular}{|c|c|c|c|c|c|}
\hline & Cuenca & $\operatorname{ADC}\left(\mathbf{k m}^{2}\right)$ & ARS $\left(\mathbf{k m}^{2}\right)$ & $\operatorname{ARI}\left(\mathbf{k m}^{2}\right)$ & $\operatorname{ARM}\left(\mathbf{k m}^{2}\right)$ \\
\hline \multirow{5}{*}{ 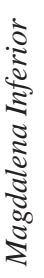 } & Magdalena inferior & 9.143 & 11.778 & 1.083 & 318 \\
\hline & Cauca inferior & 302 & 2.026 & 875 & 818 \\
\hline & Rio Cesar & 11.080 & 4.495 & 5.305 & 1.047 \\
\hline & Río Lebrija & 3.606 & 3.258 & 1.682 & 828 \\
\hline & Río San Jorge & 8.889 & 11.542 & 261 & 241 \\
\hline \multirow{6}{*}{ 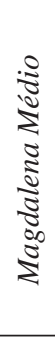 } & Magdalena medio & 3.991 & 18.686 & 21.025 & 6.162 \\
\hline & Cauca medio & $<1$ & 3.522 & 9.385 & 5.609 \\
\hline & Río Sogamoso & $<1$ & 2.458 & 652 & 0 \\
\hline & Río Suarez & $<1$ & 10.113 & 69 & $<1$ \\
\hline & Río Chicamocha & $<1$ & 8.783 & 1.016 & $<1$ \\
\hline & Río Nús & $<1$ & $<1$ & 160 & 124 \\
\hline \multirow{6}{*}{ 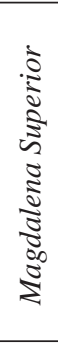 } & Magdalena superior & $<1$ & 22.410 & 16.600 & 5.658 \\
\hline & Cauca superior & 2.862 & 7.340 & 9.179 & 2.387 \\
\hline & Río Sumapaz & $<1$ & 3.293 & $<1$ & $<1$ \\
\hline & Río Neiva & $<1$ & 286 & 396 & 301 \\
\hline & Río Coello & $<1$ & 52 & 1.185 & 86 \\
\hline & Río Páez & $<1$ & 876 & 2.363 & 832 \\
\hline & TOTAL & 39.874 & 110.930 & 71.310 & 24.418 \\
\hline
\end{tabular}

ADC: área de depósitos cuaternarios, ARS: área de rocas sedimentarias, ARI: área de rocas ígneas, ARM: área de rocas metamórficas.

\section{METODOLOGÍA}

A partir del Mapa Geológico de Colombia (Gómez et al., 2007) y a los archivos digitales disponibles del IGAC (IGAC, 2017), se realizó un modelo de elevación digital (DEM) y un análisis de áreas de unidades litológicas usando ArcGIS v.10.0, para obtener el área superficial en $\mathrm{km}^{2}$ de rocas ígneas, rocas sedimentarias, rocas metamórficas y depósitos cuaternarios. Los resultados se muestran en la TABLA 1. Por otra parte, se recolectaron y analizaron al microscopio petrográfico 21 muestras de arenas recientes en barra del río Magdalena en su valle superior, medio e inferior y algunos de sus afluentes tributarios (TABLA 2). En la preparación, las muestras se sometieron a un bateo y posteriormente se utilizaron líquidos densos (LST 2,89). Los concentrados se montaron en una resina Meltmount con índice de refracción semejante al del bálsamo de Canadá de 1,54 (Mange y Maurer, 1992). El conteo fue realizado en un microscopio petrográfico en forma de bandas o "Ribbon", identificando 350 o más especies minerales por placa montada. Las muestras se ubicaron geográficamente de sur a norte y posterior a la caracterización, se realizaron asociaciones mineralógicas donde se incluyeron los minerales metaestables, inestables y ultraestables según Pettijohn et al. (1987). 1) Minerales ultraestables: aquellos minerales resistentes a procesos de disolución química (i.e. meteorización ácida), pudiendo resistir varios ciclos sedimentarios, como circón, turmalina, rutilo y anatasa. 2) Minerales metaestables: minerales moderadamente resistentes a la disolución química, generalmente resisten a no más de un ciclo sedimentario, como apatito, granate, estaurolita y monacita. 3) Minerales inestables: aquellos minerales altamente susceptibles a disolución química, generalmente en ambientes ácidos (tales como lateritas o condiciones de meteorización húmeda tropical) tienden a desaparecer, como olivino, grupo del piroxeno, grupo del anfíbol, grupo de la epidota, cianita, sillimanita, titanita, entre otros. Los resultados se encuentran en la TABLA 2. 


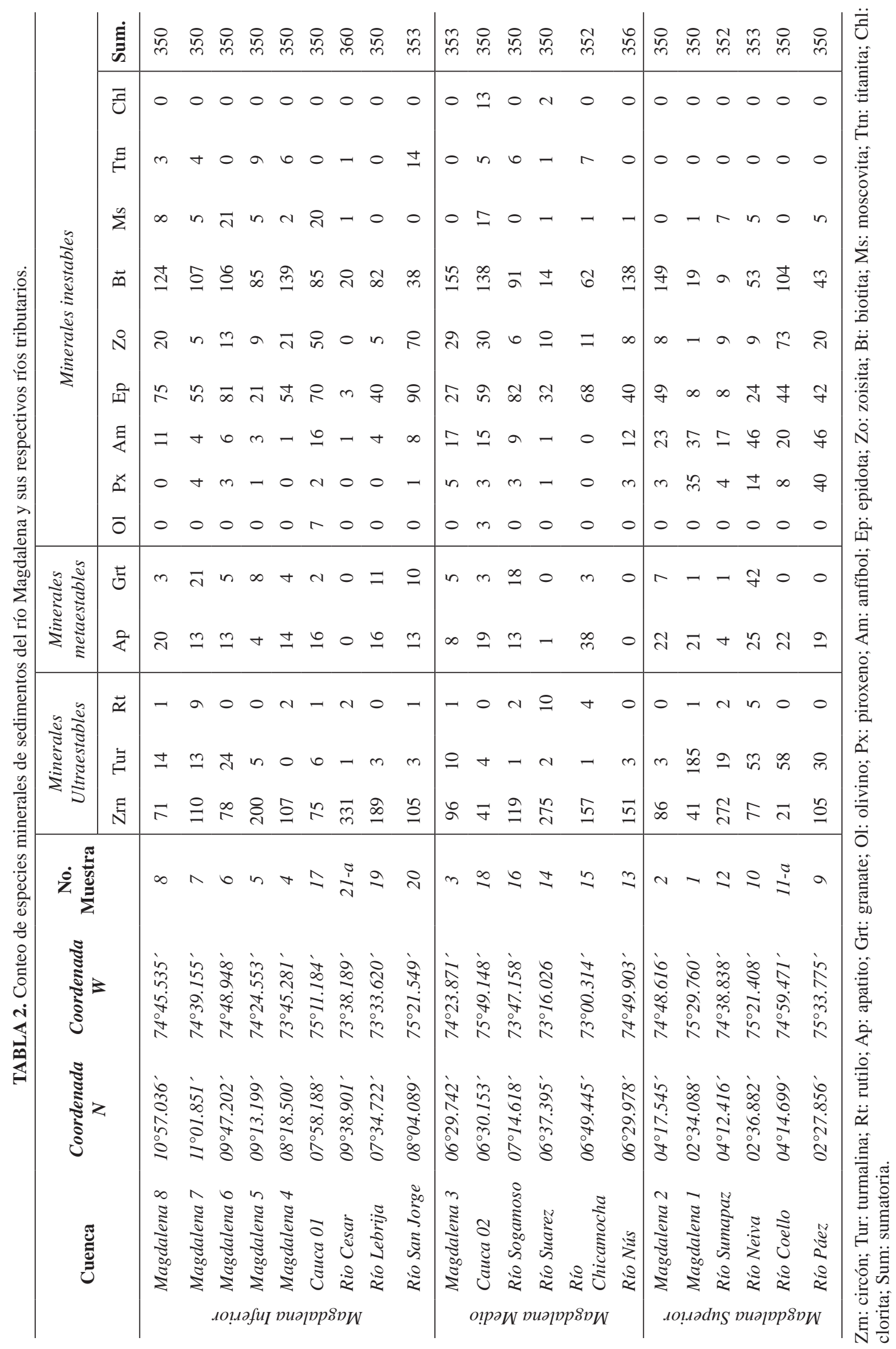




\section{RESULTADOS}

\section{Distribución espacial de las áreas fuente}

De acuerdo a la información cartográfica del Servicio Geológico de Colombia (SGC) disponible en sus plataformas digitales (Gómez et al., 2007) y al análisis espacial efectuado (TABLA 1), las rocas sedimentarias cubren la mayor parte de la Cuenca del río Magdalena, con $110.930 \mathrm{~km}^{2}$, distribuyéndose en mayor medida a lo largo de la Cordillera Oriental, la depresión Momposina y en la Serranía del Perijá, con edades que oscilan entre el Paleozoico Superior y el Mioceno. Las rocas ígneas abarcan un área de $71.310 \mathrm{~km}^{2}$, distribuidos en mayor medida a lo largo de la Cordillera Central y la vertiente oriental de la Cordillera Occidental, cuyas unidades litológicas más prominentes son las rocas plutónicas del Batolito Antioqueño y el Batolito de Ibagué, las rocas máficas del complejo Quebradagrande y la Formación Volcánica y Complejo Dagua de la Cordillera Occidental (Tello, 2001; González, 1999; Nivia, 2001; Gómez et al., 2007). Los depósitos cuaternarios cubren un área de $39.874 \mathrm{~km}^{2}$ distribuidos a lo largo del cauce del río Magdalena y su depresión Momposina. Por último, las rocas metamórficas se extienden a lo largo de $24.418 \mathrm{~km}^{2}$ principalmente en la Cordillera Central, los Macizos del norte de la Cordillera Oriental y de la Sierra Nevada de Santa Marta y la Serranía del Perijá, cuyas unidades litológicas más representativas son el Complejo Cajamarca y Arquía. Las rocas metamórficas incluyen anfibolitas, esquistos micáceos, cuarcitas y granitos de dos micas asociados, que constituyen el Complejo Cajamarca (González, 1999; Gómez et al., 2007).

\section{Caracterización y conteo de especies minerales}

La caracterización y evaluación de las asociaciones de minerales pesados se realizó por zonas. De Sur a Norte incluyen las Cordilleras Central y Occidental; la Cordillera Oriental, los macizos de Perijá y Santa Marta y el río Magdalena, normalmente, muestreado después de la llegada de los afluentes (Magdalena superior, medio e inferior, FIGURA 2). Los resultados se muestran en la TABLA 2.

\section{Sedimentos de los afluentes de las Cordilleras Central y vertiente oriental de la Cordillera Occidental}

Las especies minerales encontradas en los sedimentos de ambas cordilleras están caracterizadas por minerales inestables, cuyas especies más abundantes son minerales del grupo de la epidota $(21,6 \%)$, biotita (20\%), circón (15,4\%) y anfíbol (4,2\%) (FIGURAS 2 y 4). Los minerales menos abundantes son olivino y clorita los cuales solo se encontraron en las arenas de la Cordillera Occidental. En el conteo se pudo observar un aumento de cristales de titanita y granate en sentido Sur-Norte y una disminución en el número de cristales de turmalina (FIGURA 3). En general, los minerales inestables representan el 70\% de la media de minerales pesados a lo largo de estas dos cordilleras, mientras que los minerales ultraestables representan un 25\% y los minerales metaestables un 5\%.

\section{Sedimentos de los afluentes de la Cordillera Oriental y cuenca del río Cesar}

Las arenas de la Cordillera Oriental y de la cuenca del río Cesar se caracterizan por el predominio de circón (FIGURAS 2 y 4), con muestras cuyos contenidos de circón superan el 85\% del conteo. Otras especies ultraestables como turmalina y rutilo, con $15 \%$ y $1,4 \%$ respectivamente, tienen una abundancia restringida en las arenas del Sur de la Cordillera Oriental. Además de eso, se puede observar una disminución en el contenido de piroxeno $(0,9 \%)$ y anfíbol $(3,2 \%)$.

\section{Sedimentos del río Magdalena}

Las especies minerales más comunes obtenidas de arenas del río Magdalena son el circón (23,6\%), biotita $(20,5 \%)$ y minerales del grupo de la epidota $(9,2 \%)$ (FIGURA 2). Se puede observar que el contenido de apatito (3,07\%) es el resultado aproximado de la media de sus afluentes tributarios de la zona occidental (4\%) y la zona oriental (3,9\%). Los sedimentos muestran cierta homogeneidad Sur-Norte en su contenido de minerales inestables (50\%), metaestables (25\%) y ultraestables (25\%), exceptuando la muestra No. 1 (TABLA 2) con un notable predominio de turmalina (52,8\%). 


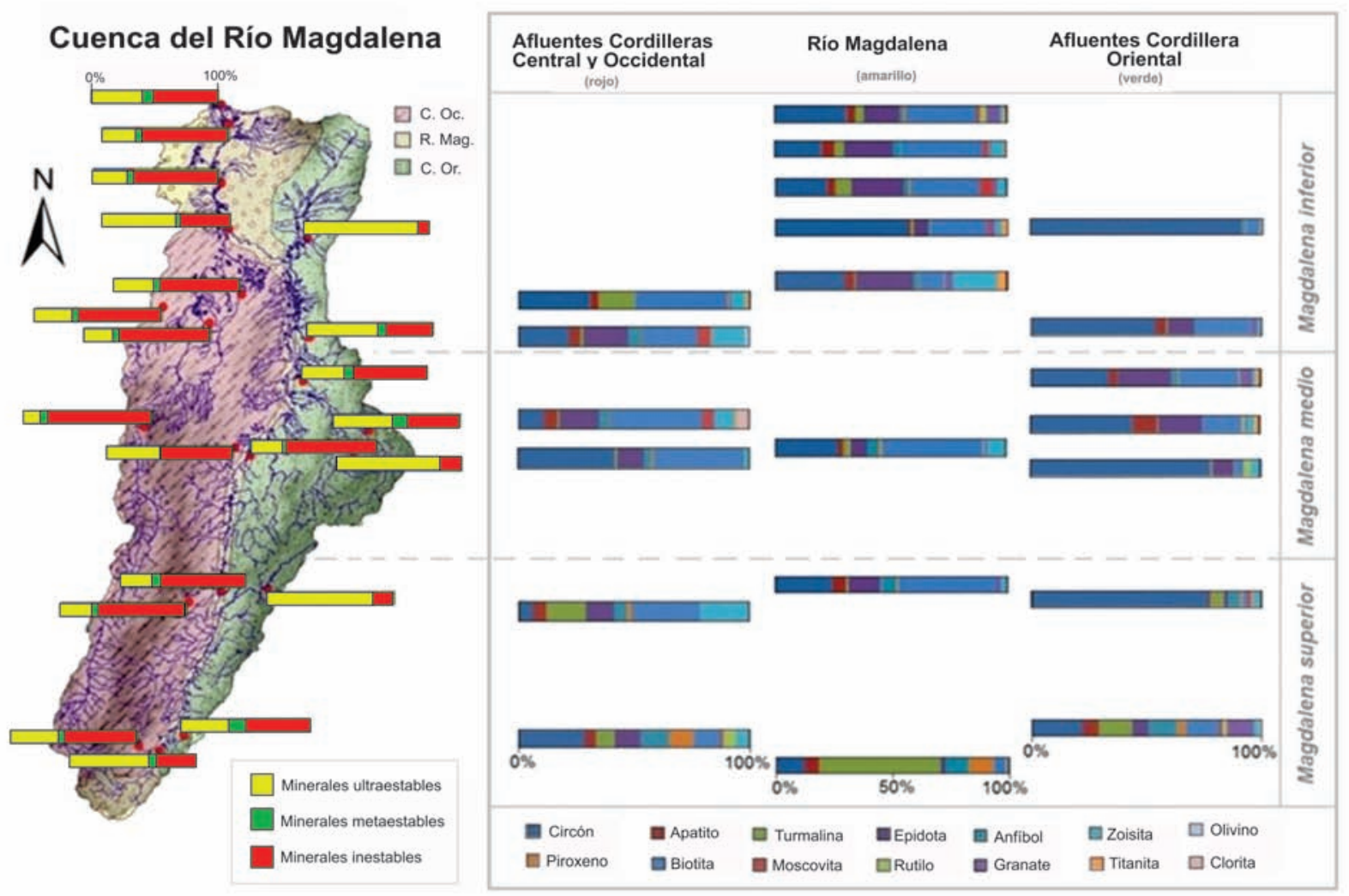

FIGURA 2. Diagramas en barra obtenidos a partir del conteo de minerales pesados y posteriormente agrupados en minerales inestables, metaestables y ultraestables, en sedimentos recientes del río Magdalena y sus ríos tributarios. C. Oc.: Cuenca Occidental; R. Mag.: Río Magdalena; C. Or.: Cuenca Oriental.

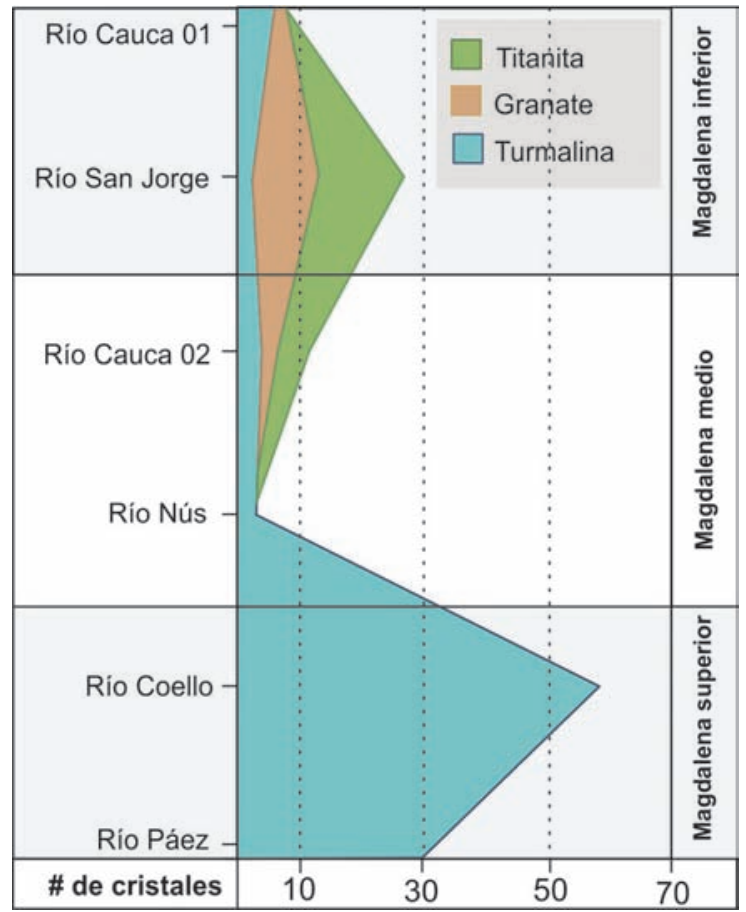

FIGURA 3. Conteo de cristales de titanita, granate y turmalina en los afluentes de las Cordilleras Central y vertiente oriental de la Cordillera Occidental, a lo largo de la cuenca del río Magdalena. 


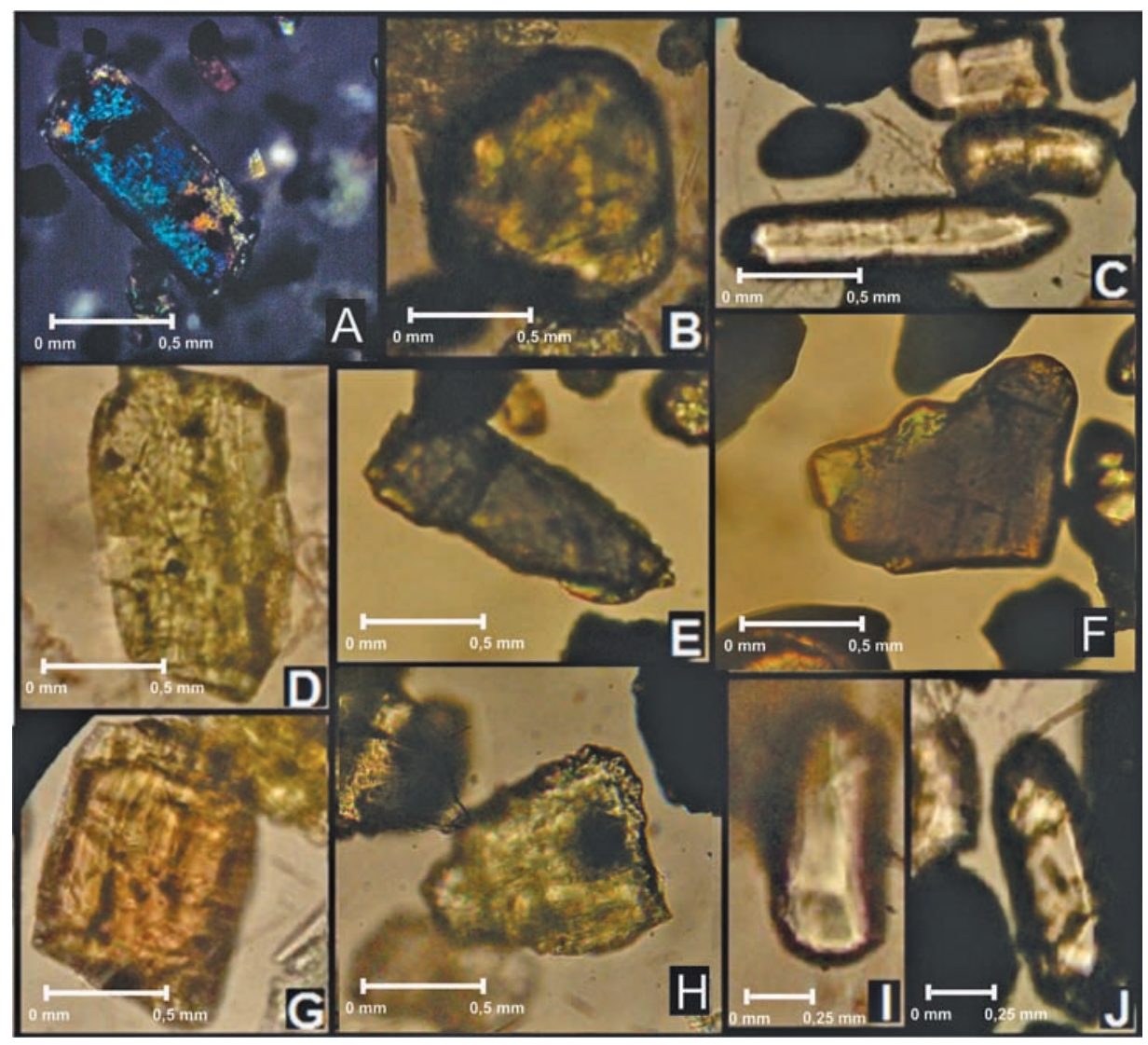

FIGURA 4. Aspecto de algunos minerales pesados del río Magdalena y sus ríos tributarios. A. Prisma de turmalina del río Magdalena 01. B. Cristal de olivino del río Cauca 02. C. Circones prismáticos del río Cesar. D. Cristal de titanita del río Magdalena 05. E. Cristal de anfíbol del río Sogamoso. F. Cristal de piroxeno del río Neiva. G. Cristal de piroxeno del río Paez. H. Grano de epidota del río Nús. I. Cristal de circón subredondeado del río Sumapaz. J. Prisma de circón subangular del río Sumapaz. Microfotografía A tomada com nicols cruzados; microfotografías B, C, D, E, F, G, H, I y J tomadas com nicols paralelos.

\section{DISCUSIÓN}

\section{Análisis de Procedencia}

\section{Drenajes de las Cordillera Central y Occidental}

De acuerdo al análisis de información geográfica (TABLA 1), con datos del SGC (Gómez et al., 2007), se observa que para la zona occidental de la Cuenca del Magdalena hay $62.512 \mathrm{~km}^{2}$ disponibles de rocas ígneas y $22.536 \mathrm{~km}^{2}$ de rocas metamórficas entre la Cordillera Central y vertiente oriental de la Cordillera Occidental. Regionalmente se encontró un predominio de minerales inestables, como biotita (25,9\%), minerales del grupo de la epidota (28,3\%) y anfíbol (5,6\%) más piroxeno $(2,7 \%)$ y olivino (0,5\%) (FIGURA 4), que podrían relacionarse con complejos volcánicos de la Cordillera Occidental y complejos ígneos y metamórficos de la Cordillera Central (González, 1999). La presencia de minerales claves en estas cuencas como olivino, piroxeno y clorita reflejan una clara basicidad del área fuente. El buen estado de preservación de las fases inestables es un resultado inesperado, debido que generalmente en climas tropicales, los procesos de meteorización química son intensos, sugiriendo por lo tanto que los sedimentos debieron tener tiempo de residencia corto en su área fuente y durante el transporte (Andò et al., 2012; Sevastjanova et al., 2012). Así, se observa que particularmente en la Cuenca del río Magdalena hay un buen estado de conservación de especies minerales inestables, y que sea pese a las condiciones de alta precipitación y temperaturas en el trópico ecuatorial, el predominio de escorrentía asociada a los significativos desniveles topográficos determinan que exista muy poco tiempo de residencia en el área fuente para que los procesos de meteorización sean realmente intensos, indicando por lo tanto que la topografía efectivamente juega un papel fundamental en la preservación de minerales inestables en climas tropicales (Sevastjanova et al., 2012). 


\section{Drenajes de la Cordillera Oriental y macizos del Perijá y Santa Marta}

La zona oriental de la Cuenca del Magdalena está constituida principalmente por secuencias sedimentarias de la Cordillera Oriental y macizos del Perijá y Santa Marta, abarcando un total de 85.560 $\mathrm{km}^{2}$ (TABLA 1). Regionalmente se observa un claro predominio de minerales ultraestables, principalmente de circón (57,4\%) (TABLA 2), resultado esperado debido a su capacidad de resistir varios ciclos sedimentarios (Pettijohn et al., 1987; Mange y Maurer, 1992), y por lo tanto reflejan el predominio de rocas sedimentarias presentes en esta cuenca. La morfología de los circones estudiados engloban desde prismas euhédricos a granos redondeados y esféricos (FIGURA 4), mostrando así granos retrabajados de diversas procedencias (Nie et al., 2012).

Biotita (13,5\%), epidota (10,5\%), apatito (3,9\%), turmalina $(3,3 \%)$ y granate $(3 \%)$ son fases comunes, aunque no tan abundantes en cuanto al circón. Otras fases minerales inestables en esta cuenca, como epidota, biotita y algunos inosilicatos, muestran un buen estado de preservación, que estarían relacionados a los diferentes macizos que exponen rocas cristalinas como el Macizo de Santander y la Sierra Nevada de Santa Marta, manteniéndose igualmente confirmada la consideración anterior sobre los efectos de la topografía en la generación de partículas inestables.

\section{Río Magdalena}

Los sedimentos del río Magdalena mostraron un alto contenido de circón $(28,1 \%)$. Los cristales de apatito (3,07\%) muestra proporciones coherentes frente a los afluentes tributarios de la zona occidental (4\%) y la zona oriental (3,9\%). Además, se observaron contenidos importantes de minerales del grupo de la epidota (9,2\%). y biotita (20,5\%) (FIGURA 2). Esto sugiere un patrón de homogenización, en el cual se diluye la proporción de minerales inestables, metaestables y ultraestables procedentes de los ríos tributarios de la zona este y oeste. La muestra "Río Magdalena 01", localizada al comienzo del valle superior, reporta un alto contenido de turmalina (52,9\%) y contenidos moderados de zircón (11,7\%), apatito (6\%), anfíbol (10,6\%) y piroxeno (10\%) (TABLA 2). Esto se puede explicar debido a que, sobre este sector, el Magdalena está empezando a desarrollar su caudal y tiene la firma geológica en los minerales pesados de las rocas plutónicas y metamórficas del Macizo de Garzón, rocas que son ricas en turmalina, como migmatitas y anatexitas (Radelli, 1962).

Como ya fue mencionado, en cuencas de clima tropical donde generalmente las reacciones químicas se producen más rápidamente, la mayoría de los minerales inestables se disuelven hasta desaparecer, incluso los minerales metaestables, aunque a tasas más lentas (Andò et al., 2012; Turner y Morton, 2007). En el caso particular de la cuenca tropical del río Magdalena, que muestra elevaciones entre 0 y 5.670 msnm (FIGURA 1), los minerales inestables son preservados ya que, con una diferencia de cota de 5.000 msnm, variables como el clima, topografía, el gradiente hidráulico y la erosión son altos, situación que difiere con las cuencas tropicales andinas de las demás cuencas tropicales ecuatoriales de geomorfología plana (Andò et al., 2012).

Finalmente, por los resultados obtenidos anteriormente, podemos observar que, en los ríos tributarios de primer y segundo orden no se modifica significativamente la composición de minerales pesados de sus cuencas, preservando generalmente de manera más precisa la composición de las rocas fuente. Por el contrario, los ríos de tercer orden como el río Magdalena, el cual es un rio troncal regional, al ser mayor receptor y mezclador de sedimentos, que incluye los minerales pesados, se enmascara la composición del área fuente, en muchos casos incrementándose la proporción de los minerales que normalmente pueden ser más abundantes, como sería el caso del circón en las regiones continentales. Adicionalmente los efectos que puede ocasionar los tiempos de residencia de la partícula durante su transporte hasta la acumulación final, como podría ser el desarrollo de barras de canal, las cuales al estar expuestas subaereamente pueden ser afectadas por procesos de meteorización, generarían una reducción de las fases más inestables.

Consideraciones sobre la influencia del orden de drenaje han sido previamente mencionadas en estudios de procedencia (Ingersoll et al., 1993), si bien el registro detrítico en sistemas de tercer orden ha sido considerado como potencial marcador del posible ambiente tectónico regional, es en los drenajes de menor orden donde realmente se preservan las características más precisas del área fuente. 


\section{CONCLUSIONES}

La zona occidental de la Cuenca del Magdalena mostró un predominio de minerales inestables como biotita (25,9\%), minerales del grupo de la epidota $(28,3 \%)$ y anfíbol $(5,6 \%)$ más piroxeno $(2,7 \%)$ y olivino $(0,5 \%)$ con buen estado de conservación, procedentes de $62.512 \mathrm{~km}^{2}$ de rocas ígneas y 22.536 $\mathrm{km}^{2}$ de rocas metamórficas de las Cordilleras Central y Occidental, lo que muestra que a escala regional las cuencas de clima tropical pueden conservar especies minerales inestables, principalmente por una alta tasa de sedimentación, alto gradiente hidráulico y alta capacidad de carga.

La zona oriental de la Cuenca del Magdalena mostró un predominio de minerales ultraestables de circón $(57,4 \%)$ y turmalina $(3,3 \%)$, procedentes de 85.560 $\mathrm{km}^{2}$ de rocas sedimentarias de la Cordillera Oriental, la Cuenca del río Cesar y Serranía del Perijá, lo que refleja positivamente su área fuente.

El mineral pesado más predominante a lo largo y ancho de la Cuenca del río Magdalena es el circón, con una media porcentual para toda la cuenca del $31,47 \%$, mostrando el potencial que puede tener la caracterización detallada de otros atributos de este mineral (geocronología U-Pb, isótopos de Hf) para la reconstrucción de la historia de procedencia, en conjunto con estudios de minerales pesados de alta resolución e índices ZTR.

Los resultados confirman que, a escala regional los minerales pesados procedentes de drenajes de primer y segundo orden pueden ser altamente diagnósticos de la geología del área fuente, mientras que los drenajes de tercer orden por su carácter mixto, tienden a obliterar las proporciones de minerales pesados, siendo por lo tanto menos diagnósticos en términos de procedencia.

\section{AGRADECIMIENTOS}

Agradecemos a Martin Pepper por facilitar el acceso a las muestras de arena, así como Camilo Bustamante y Giovanny Nova quienes también participaron en la re-colección de las mismas durante una campaña a lo largo del río Magdalena, entre Neiva y Barranquilla. Agradecemos a Gustavo Cañizales por la preparación de las muestras de minerales pesados. Finalmente agradecemos los comentarios y modificaciones sugeridas por Yamirka Rojas-Agramonte y Felipe Lamus.

\section{REFERENCIAS}

Andò, S., Garzanti, E., Padoan, M., and Limonta, M. (2012). Corrosion of heavy minerals during weathering and diagenesis: A catalog for optical analysis. Sedimentary Geology, 208, 165-178. doi: 10.1016/j.sedgeo.2012.03.023.

ESRI. (2011). ArcGIS Desktop: Release 10. Redlands, CA: Environmental Systems Research Institute. Consultado el 20 de marzo de 2014. https://www. esri.com/es-es/home.

Garzanti, E., and Andò, S. (2007a). Heavy mineral concentration in modern sands: implications for provenance interpretation. In: M.A. Mange, D.T. Wright (eds.). Developments in Sedimentology (pp. 517-545). Vol. 58, Amsterdam: Elsevier. doi: 10.1016/S0070-4571(07)58020-9.

Garzanti, E., and Andò, S. (2007b). Plate tectonics and heavy mineral suites of modern sands. In: M.A. Mange, D.T. Wright (eds.). Developments in Sedimentology (pp. 741-763). Vol. 58. Amsterdam: Elsevier. doi: 10.1016/S0070-4571(07)58029-5.

Gómez, J., Nivia, A., Montes, N.E., Jiménez, D.M., Tejada, M.L., Sepúlveda, J., Osorio, J.A., Gaona, T., Diederix, H., Uribe, H., y Mora, M. (2007). Mapa Geológico de Colombia, escala 1:2`800.000. INGEOMINAS, 2da ed., Bogotá.

González, H. (1999). Geología del departamento de Antioquia. INGEOMINAS, Bogotá. Comprende mapa a escala 1:400.000 e informe, $241 \mathrm{p}$.

IDEAM. (2001). Estudio ambiental de la Cuenca Magdalena - Cauca y elementos para su ordenamiento territorial. Bogotá: Acuerdo IDEAM-Cormagdalena.

IGAC. (2017). Sistema de Información geográfica para la planeación y el ordenamiento territorial. SIGOT. Consultado el 09 de agosto de 2017. http:// sigotn.igac.gov.co/sigotn/frames_pagina.aspx.

Ingersoll, R.V., Kretchmer, A.G., and Valles, P.K. (1993). The effect of sampling scale on actualistic sandstone petrofacies. Sedimentology, 40(5), 937953. doi: 10.1111/j.1365-3091.1993.tb01370.x. 
Leyva, P. (2001). El medio ambiente en Colombia. Bogotá: IDEAM, Ministerio del Medio Ambiente, 2da Edición.

Mange, M., and Maurer, H. (1992). Heavy Minerals in Colour. London: Chapman \& Hall.

Morton, A.C., and Hallsworth, C.R. (1999). Processes controlling the composition of heavy mineral assemblages in sandstones. Sedimentary Geology, 124(1-4), 3-29. doi: 10.1016/S00370738(98)00118-3.

Nie, J., Horton, B., Saylor, J., Mora, A., Mange, M., Garzione, C., Basu, A., Moreno, C., Caballero, V., and Parra, M. (2012). Integrated provenance analysis of a convergent retroarc foreland system: $\mathrm{U}-\mathrm{Pb}$ ages, heavy minerals, $\mathrm{Nd}$ isotopes, and sandstone compositions of the Middle Magdalena Valley basin, northern Andes, Colombia. EarthScience Reviews, 110(1-4), 111-126. doi: 10.1016/j.earscirev.2011.11.002.

Nivia, Á. (2001). Geología del departamento del Valle del Cauca. INGEOMINAS, Bogotá. Comprende mapa a escala 1:250.000 e informe, 149 p.

Pepper, M., Gehrels, G., Pullen, A., Ibanez-Mejia, M., Ward, K.M., and Kapp, P. (2016). Magmatic history and crustal genesis of western South America: Constraints from U-Pb ages and $\mathrm{Hf}$ isotopes of detrital zircons in modern rivers. Geosphere, 12(5), 1532-1555. doi: 10.1130/ GES01315.1.

Pettijohn, F.J., Potter, P.E., and Siever, R. (1987). Sand and sandstone. New York: Springer.

Radelli, L. (1962). Introducción al estudio de la petrografía del Macizo de Garzón (HuilaColombia). Geología Colombiana, 3, 17-46.
Restrepo, J. (2006). Los sedimentos del río Magdalena: reflejo de la crisis ambiental. Medellín: Fondo Editorial Universidad EAFIT.

Riezebos, P. (1979). Compositional downstream variation of opaque and translucent heavy residues in some modern Río Magdalena sands. Sedimentary Geology, 24(3-4), 197-225. doi: 10.1016/0037-0738(79)90070-8.

Sevastjanova, I., Hall, R., and Alderton, D. (2012). A detrital heavy mineral viewpoint on sediment provenance and tropical weathering in SE Asia. Sedimentary Geology, 280, 179-194. doi: 10.1016/j.sedgeo.2012.03.007.

Tello, A.N. (2001). Geología del departamento de Tolima. INGEOMINAS, Bogotá. Comprende mapa a escala 1:250.000 e informe, $101 \mathrm{p}$.

Turner, G., and Morton, A.C. (2007). The effects of burial diagenesis on detrital heavy mineral grain surface textures. In: M.A. Mange, D.T. Wright (eds.). Developments in Sedimentology (pp. 393412). Vol. 58. Amsterdam: Elsevier.

\begin{tabular}{c}
\hline Felipe Velásquez \\
ORCID: 0000-0003-1741-8588 \\
Agustín Cardona \\
ORCID: 0000-0003-2813-4820 \\
Camilo Montes \\
ORCID: 0000-0002-3553-0787 \\
\hline \hline
\end{tabular}

Trabajo recibido: octubre 15 de 2018

Trabajo aceptado: abril 08 de 2019 